initial drop in survival probability and a second drop to $18 \%$ between 20 and 40 minutes burial, where an inflection point exists and the curve levels off. Victims with an airpocket were more likely to survive compared with those without, especially for victims buried $>15$ minutes.

Conclusion.-The Austrian survival curve resembles previously published curves and suggests that underlying survival patterns are reproducible. The results are in accordance with current recommendations for management of avalanche victims and serve as a reminder that expedient companion rescue is critical for survival. For the first time an airpocket was shown to be a positive prognostic factor for survival.

\section{Medical and Logistical Challenges of the Longest Cave Rescue: A Case Report}

Thomas-Michael Schneider ${ }^{1}$; Rino Bregani ${ }^{2}$; Rok Stopar $^{3}$; Jakob Krammer ${ }^{4} ;$ Martin Göksu ${ }^{4} ;$ Natalie Müller ${ }^{1}$; Michael Petermeyer ${ }^{1}$; Johannes Schiffer ${ }^{1}$; Hermann Brugger ${ }^{5}$; Antonella Santini' ${ }^{2}$ Luca Pilo ${ }^{2}$; Giacomo Strapazzon ${ }^{5,1}$

${ }^{1}$ Bergwacht Bayern Bavarian Mountain Rescue, Bäd Tolz, Germany, ${ }^{2}$ CNSAS Italian Mountain and Cave Rescue Service, Milano, Italy, ${ }^{3}$ Mountain Rescue Association of Slovenia, Kranj, Slovenia, ${ }^{4} \ddot{O H R}$ Austrian Cave Rescue Service, Salzburg, Austria, ${ }^{5}$ EURAC Institute of Mountain Emergency Medicine, Bolzano, Italy

Introduction.- Trauma care in cave rescue is a unique situation in wilderness medicine with extremely long prehospital time. Environmental and logistical factors limit therapeutic options and transport and also can influence survival and outcome.

Objective.-To describe trauma care during the longest cave rescue reported to date.

Methods. - Case report of a 53-year-old male patient with a severe head injury at a depth of about $1000 \mathrm{~m}$ from the cave entrance in Bavaria, Germany.

Results. - Search and rescue (SAR) operation required 12 days until evacuation. SAR activation was delayed because of the location of the accident, and it required several days to recruit experienced cave rescuers and medical teams. More than 700 rescuers were involved, including 7 physicians from 5 different countries. Basic first aid was performed by companions in the acute phase and prevented the patient, who sustained a severe traumatic brain injury, from developing severe hypoxia. Thereafter, professional medical care was limited to continuous monitoring and basic therapeutic measures by medical personnel because of the extremely confined space in the cave and long evacuation time. At 6-month follow-up, the patient had recovered completely and resumed his job.

Conclusion.-First aid education and training and international collaboration of cave rescue teams play an important role in cave accidents. However, trauma care often relies on expert knowledge and is not yet evidence-based practice.

\section{A Review of Search and Rescue Operations in Slovenia}

Nicholas Walter ${ }^{1}$; Mija Gasperin ${ }^{2}$; Janina Golob Deeb ${ }^{1}$; George Deeb ${ }^{1}$ ${ }^{1}$ Virginia Commonwealth University, Richmond, VA, USA, ${ }^{2}$ General Hospital, Jesenice, Slovenia

Introduction.-Adventure recreation in wilderness areas across the world has continually increased in popularity in recent years. In response, more intensive study has been devoted to analyze and compare search and rescue programs. Furthermore, less emphasis has been put on rescue programs in smaller European countries with rich histories of mountain travel and rescue, where development of rescue systems is ongoing.

Objective.-To document trends in professional mountain rescue operations performed in Slovenia from 2011 to 2015.

Methods.-This study is a retrospective case series which uses information detailing rescue and recovery operations. Data were obtained from the Slovenian National Mountain Rescue Association. This study reports longitudinal trends in numbers of rescues and compares numbers of both on-foot and helicoptersupported rescues. We also compared data on causes of activation of search and rescue and interventions requiring use of a helicopter in 2015 and the severity of injuries requiring evacuation.

Results.-Altogether there were 434 mountain rescue operations in 2015. Among those involved, $206(42 \%)$ were injured, $176(36 \%)$ did not require treatment, $48(10 \%)$ were due to illness, and $40(8 \%)$ were found dead. The most frequent type of injury was lower extremity, and the most frequent cause of illness was exhaustion. One hundred ninety interventions (44\%) used a helicopter. Of these, $49 \%$ were injured, $29 \%$ required no medical treatment, $15 \%$ were ill, and $7 \%$ of the patients were found dead.

Conclusions. - The number of reported rescues preformed in the 5-year study period did not vary significantly despite increases in total mountain rescues throughout previous decades. A notable number of operations in the past year required the use of a helicopter, and the majority were the result of an injured patient. This study will help provide an understanding of incidents that arise in the Slovenian mountains and the response rescue services provided.

\section{How Effective Is a Dental Skills Workshop in Improv- ing the Knowledge and Confidence of Medical Students and Residents in Management of Dental Emergencies?}

Nicholas Walter; Janina Golob Deeb; Amber Johnson; George Deeb; Mikhail Bondarew

Virginia Commonwealth University, Richmond, VA, USA

Introduction.-Millions of people experience dental trauma while participating in wilderness activities. Many physicians and medical students report rarely having had any education in this field. The purpose of this study was to evaluate the effect of a 3-hour hands-on workshop for medical students and residents on their pre- and postcourse knowledge and confidence in managing dental emergencies.

Materials and Methods. - A 1-hour lecture followed by four 20-minute "hands-on" skill stations on dental mannequins was administered to a group of medical students and residents. Pre- and postworkshop questionnaire surveys were conducted.

Results.- There was a significant increase in the percent of lecture attendees who responded correctly to 3 of the 4 knowledge questions $(P<.005)$. Confidence in various statements about treating dental emergencies was significantly different after the lecture for 8 of the 9 statements. At baseline, the majority disagreed with statements implying confidence in handling various aspects of dental emergencies. At follow-up, there was an apparent trend toward confidence.

Conclusion.-Our findings suggest that dental education is minimal during undergraduate and graduate medical training. Our interactive workshop appeared to be effective in increasing the knowledge and self-reported confidence of medical practitioners in handling dental emergencies. Based on findings from this workshop, it seems there is a need for additional dental education during medical school, especially with regard to managing dental emergencies, dental anesthesia, and dental nomenclature.

\section{Battlefield Analgesia and Adherence to TCCC Guide- lines: A Quality Assurance Analysis}

Steven Schauer ${ }^{1,2}$; Andrew Fisher $^{3}$; Michael April ${ }^{2}$; Cord Cunningham ${ }^{4}$; James Aden ${ }^{1}$; Jessie Fernandez ${ }^{1}$; Robert Carter ${ }^{1}$; Robert Delorenzo ${ }^{5}$; Derek Brown ${ }^{1,2}$

${ }^{1}$ US Army Institute for Surgical Research, JBSA Fort Sam Houston, TX, USA, ${ }^{2}$ San Antonio Military Medical Center, JBSA Fort Sam Houston, TX, USA, ${ }^{3} 75$ th Ranger Regiment, Fort Benning, GA, USA, ${ }^{4} 1$ st Cavalry Division, Fort Hood, TX, USA, ${ }^{5}$ University of Texas Health Sciences Center at San Antonio, San Antonio, TX, USA

Introduction.-Previous studies have demonstrated low rates of prehospital analgesia and adherence to Tactical Combat Casualty Care (TCCC) guidelines in the Joint Theaters combat setting. The reasons for this remain unclear. We seek to expand on previous reports by evaluating a larger prehospital data set and seek associations with various factors associated with analgesia or lack of analgesia administration.

Methods.-This was part of an approved quality assurance project to evaluate adherence to TCCC guidelines across multiple modalities. Data were obtained using the Prehospital Trauma Registry (PHTR). The PHTR was in existence from January 2013 through September 2014. This database obtained data from TCCC cards, DD 1380 forms, and after-action reports (AAR) to provide real-time feedback to units on prehospital medical care.

Results.-Note that this is a preliminary analysis; full analysis will be available for the conference. During the PHTR's existence, 705 patient 